\title{
Perfusion cardiovascular magnetic resonance as the first-line technique in patients with stable chest pain
}

\author{
Monika Gawor ${ }^{1}$, Eike Nagel ${ }^{2}$ \\ 1 Department of Cardiomyopathy, National Institute of Cardiology, Warsaw, Poland \\ 2 Institute for Experimental and Translational Cardiovascular Imaging, DZHK Centre for Cardiovascular Imaging, Goethe University Hospital Frankfurt, Frankfurt am Main, Germany
}

\section{KEY WORDS \\ cardiovascular magnetic resonance, coronary artery disease, myocardial perfusion, perfusion cardiovascular magnetic resonance, stable chest pain}

\begin{abstract}
Perfusion imaging with cardiovascular magnetic resonance is a noninvasive test free of ionizing radiation recommended by the latest European Society of Cardiology guidelines as one of the functional tests for coronary artery disease (CAD) detection. It has been demonstrated in numerous studies that perfusion imaging with cardiovascular magnetic resonance is highly accurate, provide strong prognostic data, and reduce the number of unnecessary invasive angiographies in patients with stable chest pain. Implementation of this method as the first-line technique in patients with stable chest pain provides an important refinement of the current concept for the assessment of CAD and in a unique way extends the diagnostic workup beyond simply ruling in or out myocardial ischemia.
\end{abstract}

Introduction According to the 2019 European Society of Cardiology (ESC) guidelines, stable chest pain is one of the 6 most frequently encountered clinical scenarios of chronic coronary syndromes (CCS). ${ }^{1}$ However, the high rate of negative invasive coronary angiographies (ICAs) among patients with suspected coronary artery disease (CAD) demonstrates that an approach limited to ruling in or out CAD is insufficient. ${ }^{2}$ Thus, evaluation of a patient with stable chest pain should go beyond CAD assessment and instead of simply answering the question whether the patient has CAD or not, should rather provide information on the cause of patient's symptoms, the best therapeutic strategy, and finally, the patient's modifiable risk factors. Only then, the patient's prognosis can be improved, and their individual optimal therapy can be guided.

Perfusion imaging with cardiovascular magnetic resonance (perfusion-CMR) is a noninvasive test free of ionizing radiation, recommended by the latest ESC guidelines as one of the functional tests for CAD detection. ${ }^{1}$ Implementation of this method as the first-line technique in patients with stable chest pain provides an important refinement of the current concept for the assessment of patients with CAD and in a unique way extends the diagnostic workup beyond ruling in or ruling out myocardial ischemia.

Perfusion imaging with cardiovascular magnetic resonance technique For a long time, evaluation of myocardial perfusion has been a domain of nuclear imaging and stress echocardiography. Relatively recently, perfusion-CMR has become a top imaging modality for the assessment of myocardial perfusion. ${ }^{3}$ Myocardial perfusion CMR technique with adenosine- or regadenosone-induced hyperemia is a highly accurate and standardized method to detect significant CAD. ${ }^{1,3-8}$ This CMR technique is based on tracking the first pass of an intravenous bolus of gadolinium-based contrast agent (GBCA) as it circulates through the myocardium during pharmacologically obtained maximal vasodilation (stress perfusion). In the presence of a hemodynamically significant coronary lesion, myocardial hypoperfusion is observed as a subendocardial, or less frequently, as transmural, 
hypointense areas in the territory supplied by the stenotic coronary artery. Presence of a perfusion defect is usually analyzed visually. It is also possible to use one of the semiquantitative or quantitative methods of perfusion assessment. ${ }^{3}$ Interpretation of perfusion defects is performed in the context of detection of any myocardial infarction using the late gadolinium enhancement (LGE). This method is based on the assessment of myocardium in the late phase of GBCA enhancement. This sequence is usually performed 10 to 15 minutes after first pass perfusion and does not require additional injection of contrast agent. Areas of increased accumulation of GBCA (LGE areas) reflect myocardial damage, fibrosis, and infarction and provide information on myocardial viability. ${ }^{9}$ Stress perfusion images are always reviewed in comparison to LGE images. The most typical perfusion defects scenarios and corresponding LGE are shown in FIGURES 1 and 2 and Supplementary material, Figures S1 and S2. Myocardial hypoperfusion is regarded as prognostically relevant if stress perfusion defects are detected in at least 2 of 16 segments (or 4 of 32 subsegments) corresponding to an ischemic burden of more than $10 \%$ of the myocardium. In patients with previous myocardial infarction, any peri-infarct ischemia is considered as prognostically significant. ${ }^{1,10}$

For a long time, perfusion-CMR sequence was technically very difficult and prone to artefacts, because the whole data set has to be acquired every single heartbeat. To differentiate perfusion defects from artefacts, it was important to perform perfusion-CMR sequence without vasodilatation (rest perfusion). Presence of hypoperfusion in both rest- and stress-perfusion sequences with no LGE in corresponding areas suggested artefacts. Acquisition of rest perfusion sequence requires additional time and second dose of GBCA. Technical progress improved quality of stress perfusion imaging, reduced number of artefacts, and downgraded importance of rest perfusion. Current standard protocol recommended by the Society for Cardiovascular Magnetic Resonance placed rest perfusion as optional technique. ${ }^{11}$

Perfusion-CMR offers much more than assessment of myocardial ischemia. Its uniqueness lies in a comprehensive and highly accurate evaluation of patients with stable chest pain, which is not limited to the diagnostic workup of hemodynamically significant CAD. On the one hand, it allows for a more comprehensive assessment of patients with CAD and
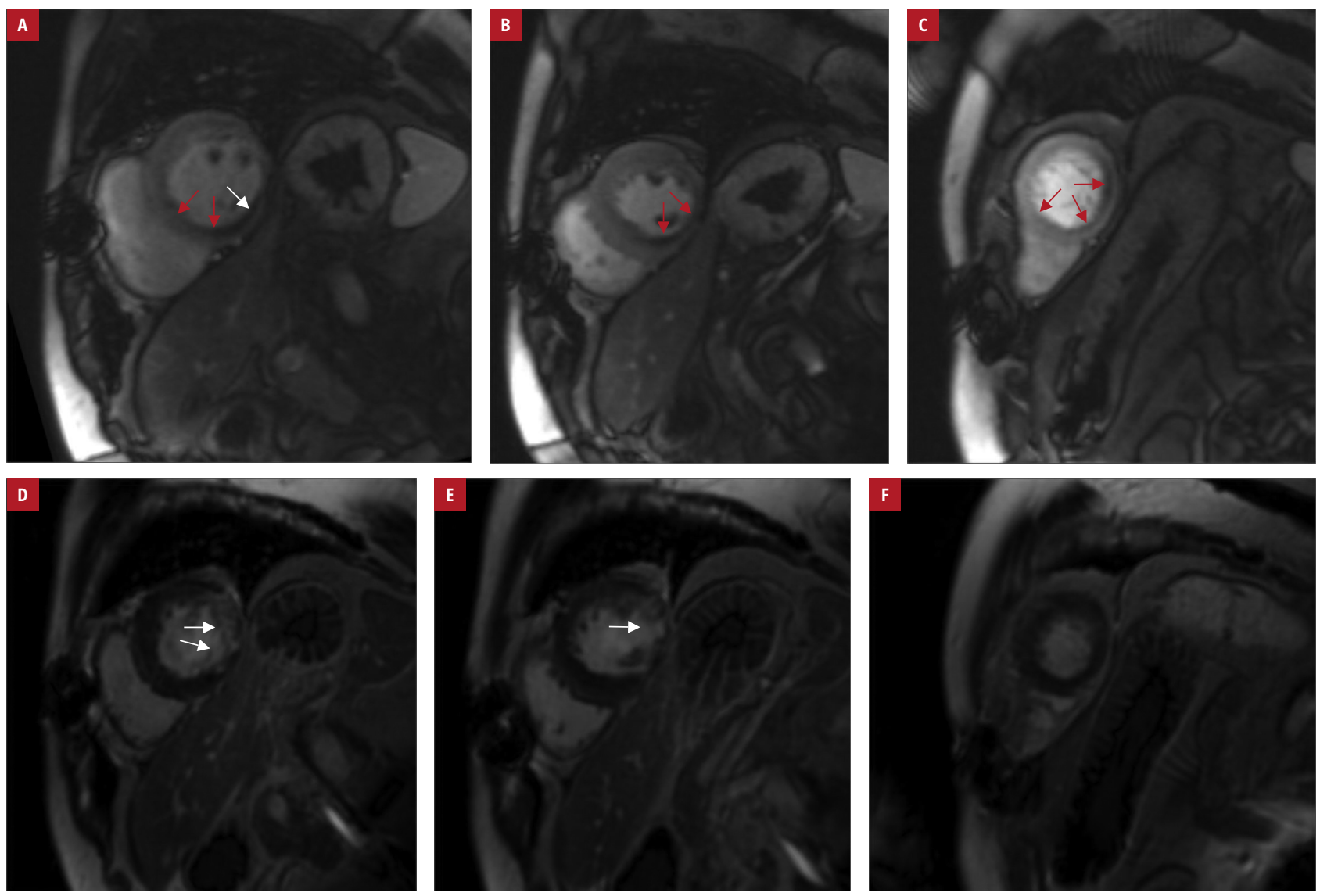

FIGURE 1 Perfusion imaging with cardiovascular magnetic resonance (CMR) during regadenoson stress in basal (A), medial (B), and apical (C) slice and corresponding CMR scar imaging (late gadolinium enhancement) in basal (D), medial (E), and apical (F) slice showing ischemic scar in the left circumflex artery territory (white arrows) and significant ischemia beyond the scar in the right coronary artery territory (red arrows) 

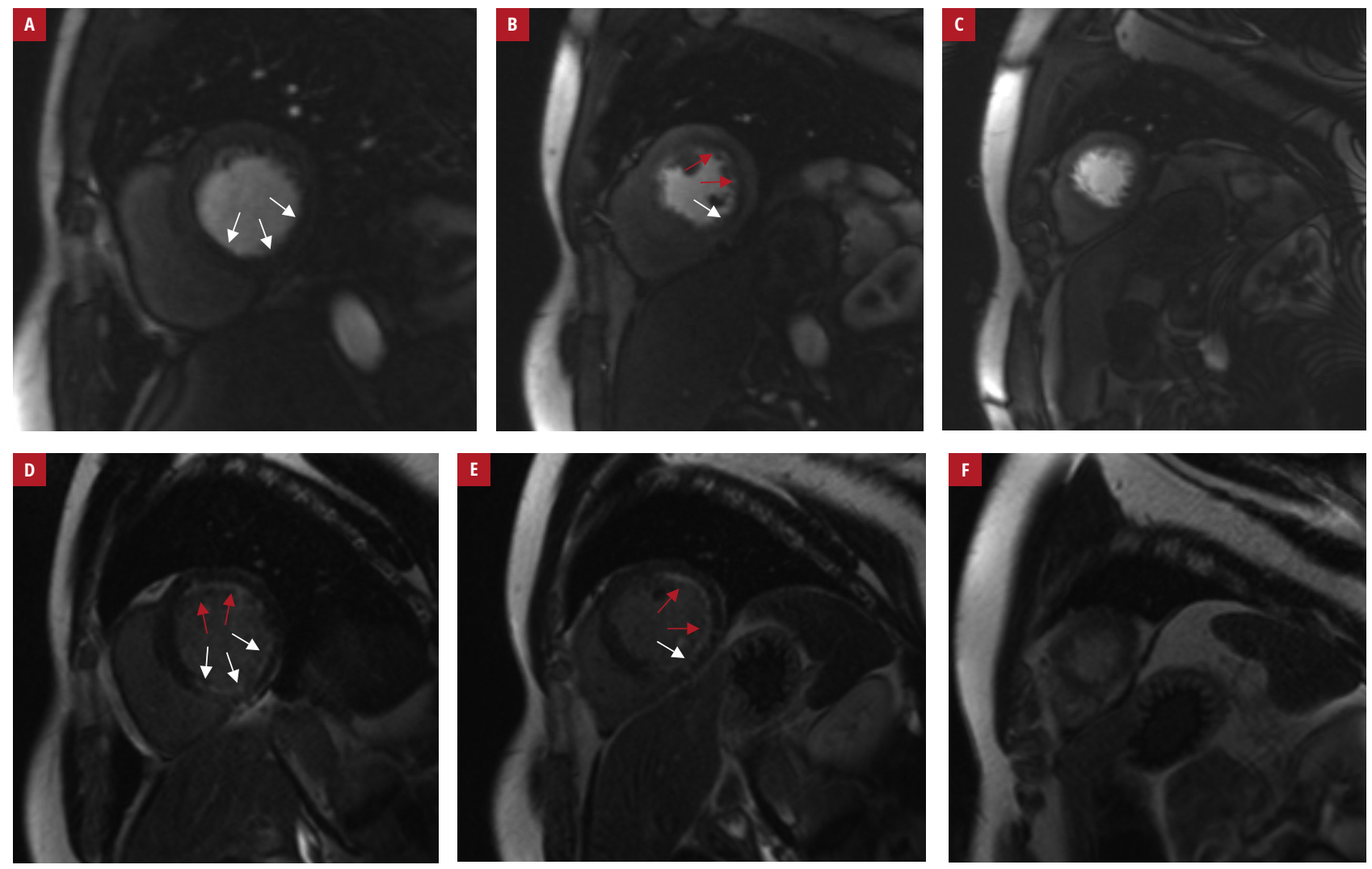

FIGURE 2 Perfusion imaging with cardiovascular magnetic resonance (CMR) during regadenoson stress in basal (A), medial (B), and apical (C) slice and corresponding CMR scar imaging (late gadolinium enhancement) in basal (D), medial (E), and apical (F) slice showing ischemic scar in the left anterior descending artery (red arrows) and right coronary artery territory (white arrows). There is no significant ischemia beyond the scar.

previous myocardial infarction. As a reference standard, CMR provides precise information on cardiac volume and function. Late gadolinium enhancement allows excellent assessment of myocardial viability. ${ }^{9}$ Novel T1 and T2 mapping techniques are extremely useful in further tissue characterization and provide information on myocardial edema, inflammation or diffuse fibrosis. ${ }^{12-18}$ Importantly, CMR hugely facilitates identification of complications related to CAD, including thrombus or postinfarction ventricular aneurysm. ${ }^{19-22}$ Accurate detection of the cardiac thrombus is important in patients undergoing stress tests, because safety of such procedure in the presence of thrombus is unknown. ${ }^{23,24}$ Perfusion-CMR allows to detect cardiac thrombus with higher sensitivity than transthoracic echocardiography. ${ }^{20,25}$ In contrary to dobutamine stress echocardiography, vasodilatators used in perfusion-CMR do not exert positive inotropic effect, which reduces potential risk of thromboembolic complications. Besides, perfusion-CMR with vasodilatators, unlike dobutamine tests, does not cause real ischemia, and thus, adverse effects due to ischemia are avoided. ${ }^{26,27}$

On the other hand, in patients with chest pain without significant coronary stenoses, perfusion-CMR in one single modality allows identification of various noncoronary pathologies and leads to the personalized management strategy. Specifically, CMR provides identification of inflammation-related diseases - such as pericarditis and myocarditis-which may cause chest pain. ${ }^{13-15}$ Perfusion-CMR enables also to explain symptoms related to microvascular obstructive disease. ${ }^{28}$ Perfusion-CMR is extremely beneficial in the assessment of patients with systemic diseases, for example lupus and diabetes, as it allows evaluation of microvascular disease as well as other abnormalities including fatty infiltration, diastolic dysfunction, and diffuse myocardial fibrosis. ${ }^{14,29}$ It may explain symptoms in patients with hypertrophic cardiomyopathy. It also helps to differentiate hypertrophic cardiomyopathy from hypertensive heart disease and provides prognostically relevant information on LGE extent. ${ }^{30-32}$ Perfusion-CMR with LGE assessment helps also to diagnose Takotsubo cardiomyopathy and allows identification of patients with myocardial infarction and nonobstructive coronary arteries. ${ }^{33,34}$ Moreover, perfusion-CMR enables detection of extracardiac findings, which also may change patient management. ${ }^{35}$

In addition to absolute contraindications to CMR (eg, non-MR safe implants), there are only a few additional conditions related to vasodilators, when perfusion-CMR cannot be performed, including sinus node disease, secondor third-degree atrioventricular ventricular 
disease. ${ }^{11}$ While adenosine may cause side effects in patients with severe asthma or severe chronic obstructive pulmonary disease, these are much less pronounced with regadenoson. In general, with both vasodilators, there has been a tendency towards raising the threshold for not performing a CMR study over the last years as the observed significant adverse events were very rare.

In comparison with ICA or computed tomography coronary angiography (CTCA), perfusion-CMR, has not only ability to identify causes of chest pain other than CAD, including pericarditis, myocarditis, or microvascular disease, but is also safer in patients with chronic kidney disease (CKD), when macrocyclic contrast agents are used. Current state of knowledge and formal recommendations for minimizing risk of nephrogenic systemic sclerosis allows the diagnostic use of macrocyclic agents in the lowest possible dose across all CKD stages. ${ }^{36,37}$ In our center (DZHK Centre for Cardiovascular Imaging, Goethe University Hospital Frankfurt, Germany), gadobutrol (Gadovist, Bayer, Leverkusen, Germany) is used at a reduced dose of $0.075 \mathrm{mmol}$ per kilogram of body weigh in all patients including those with CKD. ${ }^{5}$ Hemodialysis patients undergo dialysis on the same day that a CMR scan with GBCA was performed.

Diagnostic accuracy, prognostic value, and cost-effectiveness of perfusion imaging with cardiovascular magnetic resonance technique Recent data demonstrated that pure anatomical approach to CAD is inadequate to predict hemodynamic relevance of coronary stenoses and improved outcomes have been observed among patients undergoing functional tests. ${ }^{38-40}$ However, it remains debated which functional test is more effective in terms of accuracy, clinical outcome, and cost-effectiveness.

Perfusion-CMR has been demonstrated to be highly accurate in numerous studies, provide strong prognostic data, and reduce the number of unnecessary invasive angiographies in patients with stable chest pain. ${ }^{41-52}$ Meta-analysis, in which diagnostic accuracy of noninvasive functional tests, including perfusion-CMR has been compared with the ICA with or without fractional flow reserve (FFR) as a reference standard, showed that perfusion CMR may serve as an efficient gatekeeper to invasive assessment of CAD. ${ }^{42,46,49}$ A recent study confirmed usefulness and high diagnostic accuracy of perfusion-CMR also in stable symptomatic patients with positive coronary artery calcium score. ${ }^{53}$ Interestingly, perfusion CMR had higher diagnostic performance in 3 Tesla field compared with studies performed in 1.5 Tesla. ${ }^{48} \mathrm{~A}$ considerable amount of data, including results of 2 large randomized trials (MR-IMPACT II [Magnetic Resonance Imaging for Myocardial Perfusion Assessment in Coronary Artery Disease Trial] and CE-MARC [Cardiovascular Magnetic Resonance and Single-photon Emission Computed Tomography for Diagnosis of Coronary Heart Disease]) confirmed superiority of perfusion-CMR over single-photon emission computed tomography (SPECT) in terms of diagnostic accuracy (TABLE 1). 42-46,49,54 However, patients with implantable devices (pacemakers, implantable cardioverter-defibrillator) and significant artefacts in perfusion-CMR, may still benefit from evaluation with SPECT.

Current assessment of noninvasive imaging modalities is shifting to an evaluation of their impact on clinical outcomes rather than of their diagnostic accuracy. Based on literature data, perfusion-CMR has excellent prognostic value. ${ }^{3} \mathrm{~A}$ meta-analysis of 11636 patients with a mean follow-up of 32 months showed that negative perfusion-CMR study was associated with a very low risk of cardiovascular death and myocardial infarction. ${ }^{55}$ The results of the recent randomized clinical effectiveness trial (MR-INFORM [Myocardial Perfusion CMR versus Angiography and FFR to Guide the Management

TABLE 1 Selected studies comparing diagnostic accuracy of perfusion imaging with cardiovascular magnetic resonance versus single-photon emission computed tomography

\begin{tabular}{|c|c|c|c|c|c|}
\hline Study & Reference & $\begin{array}{l}\text { Perfusion-CMR } \\
\text { sensitivity }\end{array}$ & $\begin{array}{l}\text { Perfusion-CMR } \\
\text { specificity }\end{array}$ & SPECT sensitivity & SPECT specificity \\
\hline Jaarsma et al ${ }^{42}$ & ICA & 89 & 76 & 88 & 61 \\
\hline Greenwood et al ${ }^{44}$ & ICA & 86.5 & 83.4 & 66.5 & 82.6 \\
\hline Schwitter et al ${ }^{45}$ & ICA & 67 & 61 & 59 & 72 \\
\hline Takx et al ${ }^{46}$ & Invasive FFR & 87 & 91 & 61 & 84 \\
\hline Pontone et al ${ }^{49}$ & Invasive FFR & 81 & 91 & 64 & 82 \\
\hline
\end{tabular}

Data are presented as percentage.

Abbreviations: CMR, cardiovascular magnetic resonance; FFR, fractional flow reserve; ICA, invasive coronary angiography; SPECT, single-photon emission computed tomography 
of Patients with Stable Coronary Artery Disease]) dispelled all doubts about high prognostic value of perfusion-CMR. The study demonstrated in patients with stable angina and risk factors for CAD that perfusion-CMR is as safe and effective as ICA supported by FFR to guide the management of patients with stable chest pain with respect to the primary outcome of major adverse cardiac events at 12 months. Interestingly, the use of perfusion-CMR was associated with a significantly lower incidence of ICA and coronary revascularization than was the use of FFR-based strategy. ${ }^{5}$ Similarly, a strong prognostic power of perfusion-CMR has been demonstrated in a multicenter retrospective study, which included 2349 patients followed for a median of 5.4 years (SPINS [Stress CMR Perfusion Imaging in the United States Study]). Results of these analyses showed that patients without myocardial ischemia in CMR or LGE experienced lower incidence of cardiac adverse events, had lower need for coronary revascularization, and lower average annual cost spent on ischemia testing during follow-up. ${ }^{56} \mathrm{In}$ terestingly, first data from the ISCHEMIA (International Study of Comparative Health Effectiveness With Medical and Invasive Approaches) trial demonstrated that routine invasive therapy in comparison with optimal medical therapy do not reduce major adverse cardiac events in patients with stable ischemic heart disease and moderate to severe ischemia on noninvasive stress testing. ${ }^{57}$

Information on cost-effectiveness of perfusion-CMR are not entirely complete. While cost analysis from MR-INFORM study is still not available, previous data, including results from CE-MARC and STRATEGY (Stress Cardiac Magnetic Resonance Versus Computed Tomography Coronary Angiography for the Management of Symptomatic Revascularized Patients) study as well as from the European CMR registry, shows that using CMR is also a cost-effective strategy. ${ }^{58-60}$

Current place of perfusion imaging with cardiovascular magnetic resonance in the European Society of Cardiology guidelines Perfusion-CMR is an established noninvasive imaging method for detecting inducible myocardial perfusion deficits. The latest ESC guidelines for the diagnosis and management of CCS recommend perfusion-CMR as one of the firstline noninvasive functional tests for the assessment of CAD in symptomatic patients in whom obstructive CAD cannot be excluded by clinical evaluation alone (class IB recommendation). ${ }^{1} \mathrm{De}$ cision which initial noninvasive functional test should be selected depends on local expertise, the availability of tests, patients characteristics, and most importantly, on the clinical likelihood of CAD (class IC recommendation). Current guidelines changed the approach to the clinical assessment of CAD risk. Clinical likelihood of CAD is related to pretest probability of CAD, based on age, sex, and the nature of symptoms. However, additional features, including cardiovascular disease risk factors, abnormalities on electrocardiography and of left ventricular function, as well as coronary calcium assessed by computed tomography can further modify clinical likelihood of CAD. According to the guidelines, ICA is only indicated in a minority of cases as an alternative initial test in patients with high clinical likelihood and severe symptoms refractory to medical therapy, or typical angina at a low level of exercise and clinical evaluation that indicates high event risk (class IB recommendation). ${ }^{1}$ Functional imaging for myocardial ischemia is also recommended in cases when CTCA is not diagnostic or has shown CAD of uncertain functional significance (class IB indication). In addition, CMR may be used in low pretest likelihood patients as alternative to CTCA. ${ }^{1}$

Cardiovascular magnetic resonance versus computed tomography in perfusion imaging Recently, novel computed tomography-based methods of myocardial perfusion have emerged: stress myocardial computed tomography perfusion (stress-CTP). Stress-CTP is a newly developed technique that, if combined with CTCA, provides both anatomical and functional evaluation of CAD in a single imaging modality. Several small studies have validated this technique against the anatomical reference method (cardiac catheterization) and functional methods, including SPECT and invasive FFR, showing high sensitivity and specificity of stress-CTP in detecting flow-limiting coronary stenosis. ${ }^{46,61-63}$ A large multicenter study (CTP-PRO [Impact of Stress CT Myocardial Perfusion on Downstream Resources and Prognosis]) on the assessment of the usefulness of stress-CTP combined with CTCA in intermediate to high-risk patients for suspected CAD is still ongoing. ${ }^{64}$ Of note, there are only few studies, mostly retrospective, which compare usefulness of stress-CMR versus stress-CTP. ${ }^{65-67}$

Summary Perfusion-CMR plays a pivotal role in the work-up of patients with stable chest pain. Novel ESC guidelines for the diagnosis and management of CCS strengthen perfusion-CMR position in CAD assessment. Importantly, perfusion-CMR is useful as the first-line technique in patients with stable chest pain, replacing invasive strategy of patient management. Due to its broad diagnostic spectrum and high prognostic value, perfusion-CMR can also be implemented into diagnostic workup of patients with low clinical likelihood of CAD to exclude CAD and evaluate non-coronary pathologies responsible for chest pain. 


\section{SUPPLEMENTARY MATERIAL}

Supplementary material is available at www.mp.pl/kardiologiapolska.

\section{ARTICLE INFORMATION}

NOTE This study was presented at the 23rd International Congress of the Polish Cardiac Society; September 27, 2019; Katowice, Poland.

\section{CONFLICT OF INTEREST None declared.}

OPEN ACCESS This is an Open Access article distributed under the terms of the Creative Commons Attribution-NonCommercial-NoDerivatives 4.0 International License (CC BY-NC-ND 4.0), allowing third parties to download articles and share them with others, provided the original work is properly cited, not changed in any way, distributed under the same license, and used for noncommercial purposes only. For commercial use, please contact the journal office at kardiologiapolska@ptkardio.pl.

HOW TO CITE Gawor M, Nagel E. Perfusion cardiovascular magnetic resonance as the first-line technique in patients with stable chest pain. Kardiol Pol. 2020; 78: 98-104. doi:10.33963/KP.15204

\section{REFERENCES}

1 Knuuti J, Wijns W, Saraste A, et al. 2019 ESC guidelines for the diagnosis and management of chronic coronary syndromes. Eur Heart J Cardiovasc Imaging. 2019; 20: 1187-1197.

2 Patel MR, Peterson ED, Dai D, et al. Low diagnostic yield of elective coronary angiography. N Engl J Med. 2010; 362: 886-895.

3 Hendel RC, Friedrich MG, Schulz-Menger J, et al. CMR first-pass perfusion for suspected inducible myocardial ischemia. JACC Cardiovasc Imaging. 2016; 9: 1338-1348.

4 Ahn JH, Kim SM, Park SJ, et al. Coronary microvascular dysfunction as a mechanism of angina in severe AS: prospective adenosine-stress CMR study. J Am Coll Cardiol. 2016; 67: 1412-1422.

5 Nagel E, Greenwood JP, McCann GP, et al. Magnetic resonance perfusion or fractional flow reserve in coronary disease. N Engl J Med. 2019; 380: 2418-2428.

6 Le MTP, Zarinabad N, D'Angelo T, et al. Sub-segmental quantification of single (stress)-pass perfusion CMR improves the diagnostic accuracy for detection of obstructive coronary artery disease. J Cardiovasc Magn Reson. 2020; 22: 14.

7 Nagel E, Klein C, Paetsch I, et al. Magnetic resonance perfusion measurements for the noninvasive detection of coronary artery disease. Circulation. 2003; 108 : 432-437.

8 Al-Saadi N, Nagel E, Gross M, et al. Noninvasive detection of myocardial ischemia from perfusion reserve based on cardiovascular magnetic resonance. Circulation. 2000; 101: 1379-1383.

9 Sandstede J]. Assessment of myocardial viability by MR imaging. Eur Radiol. 2003; 13: 52-61.

10 Hachamovitch R, Hayes SW, Friedman JD, et al. Comparison of the short-term survival benefit associated with revascularization compared with medical therapy in patients with no prior coronary artery disease undergoing stress myocardial perfusion single photon emission computed tomography. Circulation. 2003; 107: 2900-2907.

11 Kramer CM, Barkhausen J, Flamm SD, et al. Standardized cardiovascular magnetic resonance (CMR) protocols 2013 update. J Cardiovasc Magn Reson. 2013; 15: 91. 12 Messroghli DR, Moon JC, Ferreira VM, et al. Clinical recommendations for cardiovascular magnetic resonance mapping of $\mathrm{T} 1, \mathrm{~T} 2, \mathrm{~T}^{*}$ and extracellular volume: A consensus statement by the Society for Cardiovascular Magnetic Resonance (SCMR) endorsed by the European Association for Cardiovascular Imaging (EACVI). J Cardiovasc Magn Reson. 2017; 19: 75.

13 Nagel E, Kwong RY, Chandrashekhar YS. CMR in nonischemic myocardial inflammation: solving the problem of diagnosing myocarditis or still diagnostic ambiguity? JACC Cardiovasc Imaging. 2020; 13: 163-166.

14 Hinojar R, Foote L, Sangle S, et al. Native T1 and T2 mapping by CMR in lupus myocarditis: Disease recognition and response to treatment. Int J Cardiol. 2016; 222: 717-726.

15 Hinojar R, Foote L, Arroyo Ucar E, et al. Native T1 in discrimination of acute and convalescent stages in patients with clinical diagnosis of myocarditis: a proposed diagnostic algorithm using CMR. JACC Cardiovasc Imaging. 2015; 8: 37-46.

16 Puntmann V0, Carr-White G, Jabbour A, et al. T1-mapping and outcome in nonischemic cardiomyopathy: all-cause mortality and heart failure. JACC Cardiovasc Imaging. 2016; 9: 40-50.

17 Puntmann V0, Carr-White G, Jabbour A, et al. Native T1 and ECV of noninfarcted myocardium and outcome in patients with coronary artery disease. J Am Coll Cardiol. 2018; 71: 766-778.

18 Śpiewak M. T1 and T2 mapping: the next giant leap in cardiac imaging. Cardiology. 2017; 138: 264-266.

19 Takasugi J, Yamagami H, Noguchi T, et al. Detection of left ventricular thrombus by cardiac magnetic resonance in embolic stroke of undetermined source. Stroke. 2017; 48: 2434-2440.

20 Bulluck H, Chan MHH, Paradies V, et al. Incidence and predictors of left ventricular thrombus by cardiovascular magnetic resonance in acute ST-segment elevation myocardial infarction treated by primary percutaneous coronary intervention: a meta-analysis. J Cardiovasc Magn Reson. 2018; 20: 72.

21 Harrity P, Patel A, Bianco J, Subramanian R. Improved diagnosis and characterization of postinfarction left ventricular pseudoaneurysm by cardiac magnetic resonance imaging. Clin Cardiol. 1991; 14: 603-606.

22 Konen E, Merchant N, Gutierrez C, et al. True versus false left ventricular aneurysm: differentiation with MR imaging-initial experience. Radiology. 2005; 236: 65-70.

23 Cusick DA, Bonow RO, Chaudhry FA. Left ventricular apical thrombus and myocardial viability: a dobutamine stress echocardiographic study. Echocardiography. 2000; 17: 547-554.

24 Cusick DA, Bonow RO, Chaudhry FA. Safety of dobutamine stress echocardiography in patients with left ventricular apical thrombus. Am J Cardiol. 1997; 80: $1252-1254$

25 Weinsaft JW, Kim J, Medicherla CB, et al. Echocardiographic algorithm for post-myocardial infarction Iv thrombus: a gatekeeper for thrombus evaluation by delayed enhancement CMR. JACC Cardiovasc Imaging. 2016; 9: 505-515.

26 Paetsch I, Jahnke C, Wahl A, et al. Comparison of dobutamine stress magnetic resonance, adenosine stress magnetic resonance, and adenosine stress magnetic resonance perfusion. Circulation. 2004; 110: 835-842.

27 Jahnke C, Nagel E, Gebker R, et al. Prognostic value of cardiac magnetic resonance stress tests: adenosine stress perfusion and dobutamine stress wall motion imaging. Circulation. 2007; 115: 1769-1776.

28 Liu A, Wijesurendra RS, Liu JM, et al. Diagnosis of microvascular angina using cardiac magnetic resonance. J Am Coll Cardiol. 2018; 71: 969-979.

29 Nagel E. Cardiovascular magnetic resonance in diabetic patients: the method of choice? Circ Cardiovasc Imaging. 2016; 9: e004699.

30 Hinojar R, Varma N, Child N, et al. T1 mapping in discrimination of hypertrophic phenotypes: hypertensive heart disease and hypertrophic cardiomyopathy: findings from the international $\mathrm{T} 1$ multicenter cardiovascular magnetic resonance study. Circ Cardiovasc Imaging. 2015; 8. pii: e003285.

31 Weng Z, Yao J, Chan RH, et al. Prognostic value of LGE-CMR in HCM: a meta-analysis. JACC Cardiovasc Imaging. 2016; 9: 1392-1402.

32 Klopotowski M, Kukula K, Malek LA, et al. The value of cardiac magnetic resonance and distribution of late gadolinium enhancement for risk stratification of sudden cardiac death in patients with hypertrophic cardiomyopathy. J Cardiol. 2016; 68: 49-56.

33 Abbas A, Sonnex E, Pereira RS, Coulden RA. Cardiac magnetic resonance assessment of takotsubo cardiomyopathy. Clin Radiol. 2016; 71: e110-e119.

34 Ferreira VM. CMR should be a mandatory test in the contemporary evaluation of "MINOCA." JACC Cardiovasc Imaging. 2019; 12: 1983-1986.

35 Dunet $V$, Barras $H$, Boulanger $X$, et al. Impact of extracardiac findings during cardiac MR on patient management and outcome. Med Sci Monit. 2015; 21: 1288-1296.

36 Reiter T, Ritter 0, Prince MR, et al. Minimizing risk of nephrogenic systemic fibrosis in cardiovascular magnetic resonance. J Cardiovasc Magn Reson. 2012; 14: 31.

37 European Medicines Agency. Gadolinium-containing contrast agents. https://www.ema.europa.eu/en/medicines/human/referrals/gadolinium-containing-contrast-agents. Accessed November 2, 2019

38 Knuuti J, Ballo H, Juarez-Orozco LE, et al. The performance of non-invasive tests to rule-in and rule-out significant coronary artery stenosis in patients with stable angina: a meta-analysis focused on post-test disease probability. Eur Heart J. 2018; 39: 3322-3330.

39 Tonino PA, Fearon WF, De Bruyne B, et al. Angiographic versus functional severity of coronary artery stenoses in the FAME study fractional flow reserve versus angiography in multivessel evaluation. J Am Coll Cardiol. 2010; 55: 2816-2821. 40 Douglas PS, Hoffmann U, Patel MR, et al. Outcomes of anatomical versus functional testing for coronary artery disease. N Engl J Med. 2015; 372: 1291-1300. 41 Schwitter J, Wacker CM, van Rossum AC, et al. MR-IMPACT: comparison of perfusion-cardiac magnetic resonance with single-photon emission computed tomography for the detection of coronary artery disease in a multicentre, multivendor, randomized trial. Eur Heart J. 2008; 29: 480-489.

42 Jaarsma C, Leiner T, Bekkers SC, et al. Diagnostic performance of noninvasive myocardial perfusion imaging using single-photon emission computed tomography, cardiac magnetic resonance, and positron emission tomography imaging for the detection of obstructive coronary artery disease: a meta-analysis. J Am Coll Cardiol. 2012; 59: 1719-1728.

43 Schwitter J, Wacker CM, Wilke N, et al. Superior diagnostic performance of perfusion-cardiovascular magnetic resonance versus SPECT to detect coronary artery disease: the secondary endpoints of the multicenter multivendor MR-IMPACT II (Magnetic Resonance Imaging for Myocardial Perfusion Assessment in Coronary Artery Disease Trial). J Cardiovasc Magn Reson. 2012; 14: 61.

44 Greenwood JP, Maredia N, Younger JF, et al. Cardiovascular magnetic resonance and single-photon emission computed tomography for diagnosis of coronary heart disease (CE-MARC): a prospective trial. Lancet. 2012; 379: 453-460.

45 Schwitter J, Wacker CM, Wilke N, et al. MR-IMPACT II: Magnetic Resonance Imaging for Myocardial Perfusion Assessment in Coronary artery disease Trial: perfusion-cardiac magnetic resonance vs. single-photon emission computed tomography for the detection of coronary artery disease: a comparative multicentre, multivendor trial. Eur Heart J. 2013; 34: 775-781. 
46 Takx RAP, Blomberg BA, El Aidi H, et al. Diagnostic accuracy of stress myocardial perfusion imaging compared to invasive coronary angiography with fractional flow reserve meta-analysis. Circ Cardiovasc Imaging. 2015; 8: e002666.

47 Greenwood JP, Ripley DP, Berry C, et al. Effect of care guided by cardiovascular magnetic resonance, myocardial perfusion scintigraphy, or NICE guidelines on subsequent unnecessary angiography rates: the CE-MARC 2 randomized clinical trial. JAMA. 2016; 316: 1051-1060.

48 Kiaos A, Tziatzios I, Hadjimiltiades S, et al. Diagnostic performance of stress perfusion cardiac magnetic resonance for the detection of coronary artery disease: a systematic review and meta-analysis. Int J Cardiol. 2018; 252: 229-233.

49 Pontone G, Guaricci AI, Palmer SC, et al. Diagnostic performance of non-invasive imaging for stable coronary artery disease: a meta-analysis. Int J Cardiol. 2020; 300: 276-281.

50 Lockie $\mathrm{T}$, Ishida M, Perera D, et al. High-resolution magnetic resonance myocardial perfusion imaging at 3.0-Tesla to detect hemodynamically significant coronary stenoses as determined by fractional flow reserve. J Am Coll Cardiol. 2011; 57: 70-75.

51 Morton G, Chiribiri A, Ishida M, et al. Quantification of absolute myocardial perfusion in patients with coronary artery disease: comparison between cardiovascular magnetic resonance and positron emission tomography. J Am Coll Cardiol. 2012; 60: 1546-1555.

52 Manka R, Wissmann L, Gebker R, et al. Multicenter evaluation of dynamic three-dimensional magnetic resonance myocardial perfusion imaging for the detection of coronary artery disease defined by fractional flow reserve. Circ Cardiovasc Imaging. 2015; 8: e003061.

53 Rijlaarsdam-Hermsen D, Lo-Kioeng-Shioe M, van Domburg RT, et al. Stress-only adenosine CMR improves diagnostic yield in stable symptomatic patients with coronary artery calcium. JACC Cardiovasc Imaging. 2020 Jan 9. [Epub ahead of print].

54 Greenwood JP, Motwani M, Maredia N, et al. Comparison of cardiovascular magnetic resonance and single-photon emission computed tomography in women with suspected coronary artery disease from the Clinical Evaluation of Magnetic Resonance Imaging in Coronary Heart Disease (CE-MARC) trial. Circulation. 2014; 129: 1129-1138.

55 Lipinski M], McVey CM, Berger JS, et al. Prognostic value of stress cardiac magnetic resonance imaging in patients with known or suspected coronary artery disease: a systematic review and meta-analysis. J Am Coll Cardiol. 2013; 62: $826-838$

56 Kwong RY, Ge Y, Steel K, et al. Cardiac magnetic resonance stress perfusion imaging for evaluation of patients with chest pain. J Am Coll Cardiol. 2019; 74: $1741-1755$.

57 American College of Cardiology. International Study of Comparative Health Effectiveness With Medical and Invasive Approaches - ISCHEMIA. https://www.acc.org/latest-in-cardiology/clinical-trials/2019/11/15/17/27/ ischemia\#treferences-for-article. Accessed February 9, 2020.

58 Walker S, Girardin F, McKenna C, et al. Cost-effectiveness of cardiovascular magnetic resonance in the diagnosis of coronary heart disease: an economic evaluation using data from the CE-MARC study. Heart. 2013; 99: 873-881.

59 Moschetti K, Petersen SE, Pilz G, et al. Cost-minimization analysis of three decision strategies for cardiac revascularization: results of the "suspected CAD" cohort of the European cardiovascular magnetic resonance registry. J Cardiovasc Magn Reson. 2016; 18: 3

60 Pontone G, Andreini D, Guaricci AI, et al. The STRATEGY study (Stress Cardiac Magnetic Resonance Versus Computed Tomography Coronary Angiography for the Management of Symptomatic Revascularized Patients): resources and outcomes impact. Circ Cardiovasc Imaging. 2016; 9: e005171.

61 Yang J, Dou G, He B, et al. Stress myocardial blood flow ratio by dynamic CT perfusion identifies hemodynamically significant CAD. JACC Cardiovasc Imaging. 2019 Sep 12. [Epub ahead of print].

62 Ueki Y, Izawa A, Kashiwagi D, et al. Diagnostic advantage of stress computed tomography myocardial perfusion over single-photon emission computed tomography for the assessment of myocardial ischemia. J Cardiol. 2017; 70: 147-154.

63 Okada DR, Ghoshhajra BB, Blankstein R, et al. Direct comparison of rest and adenosine stress myocardial perfusion CT with rest and stress SPECT. J Nucl Cardiol. 2010; 17: 27-37.

64 Pontone G, De Cecco C, Baggiano A, et al. Design of CTP-PRO study (impact of stress Cardiac computed Tomography myocardial Perfusion on downstream resources and PROgnosis in patients with suspected or known coronary artery disease: a multicenter international study). Int J Cardiol. 2019; 292: 253-257.

65 Tanabe Y, Kido T, Uetani T, et al. Differentiation of myocardial ischemia and infarction assessed by dynamic computed tomography perfusion imaging and comparison with cardiac magnetic resonance and single-photon emission computed tomography. Eur Radiol. 2016; 26: 3790-3801.

66 Chung HW, Ko SM, Hwang HK, et al. Diagnostic performance of coronary CT angiography, stress dual-energy CT perfusion, and stress perfusion single-photon emission computed tomography for coronary artery disease: comparison with combined invasive coronary angiography and stress perfusion cardiac MRI. Korean J Radiol. 2017; 18: 476-486.

67 Oleksiak A, Kruk M, Pugliese F, et al. Regadenoson dynamic computed tomography myocardial perfusion using low-dose protocol for evaluation of the ischemic burden. ULYSSES study. J Cardiovasc Comput Tomogr. 2020 Jan 21. [Epub ahead of print]. 